\title{
A RESULT CONCERNING ADDITIVE FUNCTIONS IN HERMITIAN BANACH *-ALGEBRAS AND AN APPLICATION
}

\author{
J. VUKMAN
}

\begin{abstract}
Let $A$ be a complex hermitian Banach ${ }^{*}$-algebra with an identity element $e$. Suppose there exists an additive function $f: A \rightarrow A$ such that $f(a)=-a^{*} a f\left(a^{-1}\right)$ holds for all normal invertible elements $a \in A$. We prove that in this case $f$ is of the form $f(a)=f(i e) k$, where $a=h+i k$. Using this result we generalize S. Kurepa's extension of Jordan-Neumann characterization of pre-Hilbert space.
\end{abstract}

This research has been inspired by the work of S. Kurepa $[\mathbf{2}, \mathbf{3}]$ and P. Vrbová [6]. All algebras and vector spaces in this paper will be over the complex field. Algebras are assumed to have an identity element, which will be denoted by $e$. An algebra $A$ is called a ${ }^{*}$-algebra if there exists an involution (conjugate-linear antiisomorphism of period two) $a \mapsto a^{*}$ on $A$. An element $h \in A$ is said to be hermitian if $h^{*}=h$, and $u \in \mathcal{A}$ is said to be unitary if $u^{*} u=u u^{*}=e$. An element $a \in \mathcal{A}$ will be called normal if $a^{*} a=a a^{*}$. It is easy to see that each element $a \in A$ has a unique decomposition $a=h+i k$ with hermitian $h$ and $k$. An element $a \in A$ is normal if and only if $h$ and $k$ commute.

A *-algebra which is also a Banach algebra is called a Banach *-algebra. A Banach *-algebra is called hermitian if each hermitian element has real spectrum. Let $A$ be a hermitian Banach *-algebra and let $h \in A$ be a hermitian element. It is convenient to write $h>0(h \geq 0)$ if the spectrum of $h$ is positive (nonnegative). The notation $h>k(h \geq k)$ means $h-k>0(h-k \geq 0)$. The most important hermitian Banach *-algebras are $B^{*}$-algebras (i.e. Banach ${ }^{*}$-algebras in which $\left\|a^{*} a\right\|=\|a\|^{2}$ is fulfilled for all $a$ ). For basic facts concerning hermitian Banach *-algebras, we refer the reader to $\mathrm{V}$. Pták's paper [5].

Let $X$ and $A$ be a vector space and an algebra, respectively. Suppose that $X$ is a left $A$-module. A left $A$-module $X$ will be called unitary if $e x=x$ holds for all $x \in X$, and will be called irreducible if for each pair $x, y \in X, x \neq 0$, there exists $a \in A$ such that $a x=y$.

First we shall consider the following result.

THEOREM 1. Let $A$ be a hermitian Banach ${ }^{*}$-algebra. Suppose there exists an additive function $f: A \rightarrow A$ such that $f(a)=-a^{*} a f\left(a^{-1}\right)$ holds for all normal invertible elements $a \in A$. In this case $f(a)=f(i e) k$ is fulfilled for all $a=h+i k$.

REMARK. If $A$ is the complex number field, then the theorem above reduces to a result due to $\mathrm{P}$. Vrbová [6].

Received by the editors June 7, 1983 and, in revised form, September 15, 1983.

1980 Mathematics Subject Classification. Primary 46K05, 46L05.

Key words and phrases. *-algebra, Banach *-algebra, hermitian Banach *-algebra, $B^{*}$-algebra, vector space, module, additive function, $A$-bilinear form, $A$-quadratic form. 
For the proof of Theorem 1 we need the lemma below. We omit the proof since it is an easy consequence of Ford's square root lemma [1 or $\mathbf{5},(1.5)]$.

LEMMA 2. Let $A$ be a hermitian Banach ${ }^{*}$-algebra. For each $h>0$ there corresponds $u>0$, such that $u^{2}=h$. Moreover, $u$ commutes with each element which commutes with $h$.

PROOF OF THEOREM 1. Let us first prove that

$$
f(h)=0
$$

holds for all $h \in A, 0<h<e$. Since in this case $e-h^{2}>0$, there exists, by Lemma 2, a hermitian element $k$, such that $k$ commutes with $h$, and $e-h^{2}=k^{2}$, whence it follows that $u=h+i k$ is a unitary element. Therefore, according to the requirements of the theorem, we have

$$
f(h)+f(i k)=f(u)=-u^{*} u f\left(u^{-1}\right)=-f(h-i k)=-f(h)+f(i k),
$$

whence it follows $f(h)=0$. Let us prove that

$$
f(t e)=0
$$

for each real number $t$. If $0 \leq t<1$, then (2) follows from (1). It is easy to see that $f(e)=0$. Therefore (2) holds for all $t \in[0,1]$. If $t>1$, we have $0<t^{-1}<1$, whence $f(t e)=-t^{2} f\left(t^{-1} e\right)=0$, which proves that (2) holds for all nonnegative real numbers and therefore also for all real numbers. Let us prove that

$$
f(h)=0
$$

for all hermitian $h \in \mathcal{A}$. Therefore, let $h$ be an arbitrary hermitian element, and let us choose a real number $t$ such that $t e+h>e$. Then $0<(t e+h)^{-1}<e$. According to $(1)$ we have $f(t e+h)=-(t e+h)^{2} f\left((t e+h)^{-1}\right)=0$. Hence $f(h)=f(-t e)$ and, according to $(2), f(h)=0$. Now we intend to prove that

$$
f(i h)=h f(i e)
$$

holds for all $h \in A, 0<h<e$. From $0<h<e$ it follows that $h-h^{2}>0$. By Lemma 2 there exists a hermitian element $k$, such that $k$ commutes with $h$, and that $h-h^{2}=k^{2}$. The element $a=k+i h$ is normal, since $h$ and $k$ commute. Since $a$ can be expressed in the form $a=h\left(h^{-1} k+i e\right)$, it is obvious that $a$ is invertible (recall that $A$ is by assumption hermitian). Therefore using the requirements of the theorem and (3) we obtain

$$
\begin{aligned}
f(i h) & =f(k)+f(i h)=f(a)=-a^{*} a f\left(a^{-1}\right)=-a^{*} a f\left(\left(a^{*} a\right)^{-1} a^{*}\right) \\
& =-\left(h^{2}+k^{2}\right) f\left(\left(h^{2}+k^{2}\right)^{-1}(k-i h)\right)=-h f\left(h^{-1}(k-i h)\right) \\
& =-h f\left(h^{-1} k\right)+h f(i e)=h f(i e) .
\end{aligned}
$$

Let us prove that

$$
f(i t e)=t f(i e)
$$

holds for each real number $t$. If $0 \leq t \leq 1$, then (5) follows from (4). If $t>1$, then $0<t^{-1}<1$, and we have

$$
f(i t e)=-t^{2} f\left((i t e)^{-1}\right)=-t^{2} f\left(-i t^{-1} e\right)=t^{2} t^{-1} f(i e)=t f(i e)
$$


which proves that (5) holds for all nonnegative real numbers and therefore also for all real numbers. We shall prove that

$$
f(i h)=h f(i e)
$$

is fulfilled for each hermitian element $h \in A$. Therefore, let $h$ be an arbitrary hermitian element, and let us choose a real number $t$ such that $t e+h>e$. Then $0<(t e+h)^{-1}<e$. According to (4) we have

$$
\begin{aligned}
f(i(t e+h)) & =-(t e+h)^{2} f\left((i(t e+h))^{-1}\right) \\
& =(t e+h)^{2}(t e+h)^{-1} f(i e)=(t e+h) f(i e) .
\end{aligned}
$$

Using the additivity of the function $f$ and (5), we obtain $f(i h)=h f(i e)$. From (3), (6) and the fact that each $a \in A$ can be expressed in the form $a=h+i k$, where $h$ and $k$ are hermitian, it follows $f(a)=k f(i e)$, which completes the proof of the theorem.

Let $X$ and $A$ be a complex vector space and a complex *-algebra, respectively. Suppose that $X$ is a left $A$-module. A mapping $B(\cdot, \cdot): X \times X \rightarrow A$ is called an $A$-bilinear form if

$1^{\circ} B\left(a_{1} x_{1}+a_{2} x_{2}, y\right)=a_{1} B\left(x_{1}, y\right)+a_{2} B\left(x_{2}, y\right), x_{1}, x_{2}, y \in X, a_{1}, a_{2} \in \mathcal{A}$,

$2^{\circ} B\left(x, a_{1} y_{1}+a_{2} y_{2}\right)=B\left(x, y_{1}\right) a_{1}^{*}+B\left(x, y_{2}\right) a_{2}^{*}, x, y_{1}, y_{2} \in X, a_{1}, a_{2} \in \mathcal{A}$.

A mapping $Q: X \rightarrow A$ is called an $A$-quadratic form if

$3^{\circ} Q(x+y)+Q(x-y)=2 Q(x)+2 Q(y), x, y \in X$,

$4^{\circ} Q(a x)=a Q(x) a^{*}, x \in X, a \in \mathcal{A}$.

Let us consider two examples of $A$-bilinear forms.

EXAMPLE 1. Let $A$ be a *-algebra and $\mathcal{L} \subset A$ a left ideal. Considering $\mathcal{L}$ as a left $A$-module, one can introduce an $A$-bilinear form $B(\cdot, \cdot)$ as follows $B(x, y)=$ $x y^{*}, x, y \in \mathcal{L}$.

EXAMPLE 2. Let $X$ be a Hilbert space and let us denote by $L(X)$ the algebra of all bounded linear operators of $X$ into itself. Let the involution on $L(X)$ be the adjoint operation. $X$ can be considered as a unitary irreducible left $L(X)$-module (multiplication by $A \in L(X)$ is operator action on $X$ ). A simple calculation shows that the mapping $B(\cdot, \cdot): X \times X \rightarrow L(X)$ defined by the relation $B(x, y) z=(z, y) x$, where $(\cdot, \cdot)$ denotes the inner product in $X$, is an $A$-bilinear form.

It is easy to see that each $A$-bilinear form gives rise to the $A$-quadratic form by the relation $Q(x)=B(x, x)$. It seems natural to ask whether the converse is also true. More precisely, we consider the following

PROBLEM. Let $X$ and $A$ be a vector space and a *-algebra, respectively. Suppose that $X$ is a left $\mathcal{A}$-module, and that there exists an $A$-quadratic form $Q: X \rightarrow \mathcal{A}$. Does there exist an $A$-bilinear form $B(\cdot, \cdot): X \times X \rightarrow A$ such that $Q(x)=B(x, x)$ holds for all $x \in X$ ?

It follows from a result of S. Kurepa [3] that the answer to the question above is affirmative if $A$ is the complex number field. His result can be formulated as follows.

THEOREM 3 (S. KUREPA [3]). Let $X$ be a vector space over the complex field $C$. Suppose there exists a mapping $Q: X \rightarrow C$ such that $Q(x+y)+Q(x-y)=$ $2 Q(x)+2 Q(y), Q(\lambda x)=|\lambda|^{2} Q(x)$ holds for all pairs $x, y \in X$ and all $\lambda \in C$. Under these conditions the mapping $B(\cdot, \cdot): X \times X \rightarrow C$ defined by

$$
B(x, y)=\frac{1}{4}(Q(x+y)-Q(x-y))+\frac{i}{4}(Q(x+i y)-Q(x-i y))
$$


is additive in both arguments, and $B(\lambda x, y)=\lambda B(x, y), B(x, \lambda y)=\bar{\lambda} B(x, y)$ hold for all pairs $x, y \in X$ and all $\lambda \in C$. For each $x \in X$ the relation $Q(x)=B(x, x)$ is fulfilled.

REMARKS. The theorem above can be considered as an extension of the wellknown result due to P. Jordan and J. von Neumann which characterizes pre-Hilbert space among all normed spaces. It should be mentioned that P. Vrbová [6] has obtained a simple proof of S. Kurepa's theorem. Using Theorem 1 and an approach from [6] we prove the result below which can be considered as a generalization of Theorem 3.

THEOREM 4. Let $X$ be a vector space and $A$ a commutative hermitian Banach *-algebra. Let $X$ be a unitary $A$-module, and suppose that there exists an $A$-quadratic form $Q: X \rightarrow A$. In this case the mapping $B(\cdot, \cdot): X \times X \rightarrow A$ defined by

$$
B(x, y)=\frac{1}{4}(Q(x+y)-Q(x-y))+\frac{i}{4}(Q(x+i y)-Q(x-i y))
$$

is an $A$-bilinear form. For all $x \in X$ the relation $Q(x)=B(x, x)$ holds.

PROOF. Let us first prove that the function $S(\cdot, \cdot)$ defined by the relation $S(x, y)=Q(x+y)-Q(x-y)$ is additive in both variables. This part of the proof goes through as in the proof of Theorem 3 (see [3] and also [4] for some generalizations), but we shall write it down for the sake of completeness. It is easy to see that $Q(0)=0$ and $Q(-x)=Q(x), x \in X$. For arbitrary elements $x, y, u \in X$ we have

$$
\begin{aligned}
S(x+y, 2 u)= & Q(x+y+2 u)-Q(x+y-2 u) \\
= & Q((x+u)+(y+u))+Q((x+u)-(y+u)) \\
& -Q((x-u)+(y-u))-Q((x-u)-(y-u)) .
\end{aligned}
$$

Using the relation $Q(x+y)+Q(x-y)=2 Q(x)+2 Q(y)$ we obtain

$$
\begin{aligned}
S(x+y, 2 u) & =2 Q(x+u)+2 Q(y+u)-(2 Q(x-u)+2 Q(y-u)) \\
& =2 S(x, u)+2 S(y, u) .
\end{aligned}
$$

Hence

$$
S(x+y, 2 u)=2 S(x, u)+2 S(y, u) .
$$

Putting $y=0, x=z$ we obtain $S(z, 2 u)=2 S(z, u)$. Substituting $z$ by $x+y$ and using (7) we finally obtain

$$
2 S(x+y, u)=S(x+y, 2 u)=2 S(x, u)+2 S(y, u)
$$

which proves that the function $S(\cdot, \cdot)$ is additive in the first variable. Since $S(x, y)=$ $S(y, x)$ for all pairs $x, y \in X$ (this follows from the relation $Q(-x)=Q(x)$ ) it follows that the function $S(\cdot, \cdot)$ is additive also in the second variable. From the fact that $S(\cdot, \cdot)$ is additive in both variables, it follows that the same is true for the function $B(\cdot, \cdot)$ defined by the relation $B(x, y)=\frac{1}{4} S(x, y)+\frac{i}{4} S(x, i y)$. Therefore, since it is easy to see that $Q(x)=B(x, x)$ holds for all $x \in X$, it remains to prove that

$$
B(a x, y)=a B(x, y), \quad B(x, a y)=a^{*} B(x, y)
$$


is fulfilled for all pairs $x, y \in X$ and all $a \in A$. Now we are going to use the condition $Q(a x)=a^{*} a Q(x)$. First of all it follows from the condition above that

$$
S(a x, y)=a^{*} a S\left(x, a^{-1} y\right)
$$

holds for all pairs $x, y \in X$ and all invertible $a \in A$. Let us prove that $B(\cdot, \cdot)$ satisfies the relations

$$
\begin{aligned}
& B(i x, y)=i B(x, y) \\
& B(x, i y)=-i B(x, y)
\end{aligned}
$$

Indeed,

$$
\begin{aligned}
4 B(i x, y) & =S(i x, y)+i S(i x, i y)=S(x,-i y)+i S(x, y) \\
& =i(S(x, y)-i S(x,-i y))=i(S(x, y)+i S(x, i y))=4 i B(x, y)
\end{aligned}
$$

which proves (10). Furthermore,

$$
\begin{aligned}
4 B(x, i y) & =S(x, i y)+i S(x,-y)=S(x, i y)-i S(x, y) \\
& =-i(S(x, y)+i S(x, i y))=-4 i B(x, y) .
\end{aligned}
$$

Now we intend to prove that for the function $f: A \rightarrow A$, defined by the relation

$$
f(a)=B(a x, y)-B(x, a y),
$$

where $x$ and $y$ are fixed vectors, the requirements of Theorem 1 are fulfilled. Since the additivity of the function above follows from the fact that $B(\cdot, \cdot)$ is additive in both variables, it remains to show that $f(a)=-a^{*} a f\left(a^{-1}\right)$ holds for all invertible $a \in A$. We have

$$
\begin{aligned}
4 f(a) & =S(a x, y)+i S(a x, i y)-(S(x, a y)+i S(x, i a y)) \\
& =S(a x, y)+i S(a x, i y)-(S(a y, x)+i S(i a y, x))
\end{aligned}
$$

Using (9) we obtain

$$
\begin{aligned}
4 f(a) & =a^{*} a\left(S\left(x, a^{-1} y\right)+i S\left(x, i a^{-1} y\right)\right)-a^{*} a\left(S\left(y, a^{-1} x\right)+i S\left(i y, a^{-1} x\right)\right) \\
& =4 a^{*} a\left(B\left(x, a^{-1} y\right)-B\left(a^{-1} x, y\right)\right)=-4 a^{*} a f\left(a^{-1}\right) .
\end{aligned}
$$

According to Theorem 1 we have $f(h+i k)=f(i e) k$ for all hermitian $h$ and $k$. In particular, $f(h)=0$ which implies

$$
B(h x, y)=B(x, h y)
$$

for all hermitian $h \in A$ and all pairs $x, y \in X$. If we put $a=i h, h$ hermitian, we obtain

$$
B(i h x, y)-B(x, i h y)=f(i h)=h f(i e)=h(B(i x, y)-B(x, i y)) .
$$

Using (10), (11) and (13) we obtain

$$
B(h x, y)=h B(x, y) \text {. }
$$

Therefore according to (10), (11), (13) and (14) it follows that (8) holds. The proof of the theorem is complete.

REMARK. It would be interesting to know whether Theorem 4 holds also in the noncommutative case.

We conclude with the following purely algebraic result. 
THEOREM $5 \cdot^{-}$Let $X$ be a vector space and $A$ a commutative *-algebra. Let $X$ be $a$ unitary irreducible $A$-module, and suppose that there exists an $A$-quadratic form $Q: X \rightarrow A$. In this case the mapping $B(\cdot, \cdot): X \times X \rightarrow A$ defined by

$$
B(x, y)=\frac{1}{4}(Q(x+y)-Q(x-y))+\frac{i}{4}(Q(x+i y)-Q(x-i y))
$$

is an $A$-bilinear form. For all $x \in X$ the relation $Q(x)=B(x, x)$ holds.

PROOF. It remains to prove that

$$
S(h x, y)=h S(x, y), \quad S(x, h y)=h S(x, y),
$$

where $S(x, y)$ stands for $Q(x+y)-Q(x-y)$, holds for all pairs $x, y \in X$ and each hermitian $h \in A$, since the rest of the proof goes through as in the proof of Theorem 4. Therefore, let $x, y \in X, h \in \mathcal{A}, h^{*}=h$ be arbitrary, and let us prove (15). We may assume that $x \neq 0$, since there is nothing to prove if $x=y=0$. By the requirements of the theorem there exists $a \in \mathscr{A}$ such that $y=a x$. We have

$$
\begin{aligned}
S(h x, y) & =S(h x, a x)=Q((h+a) x)-Q((h-a) x) \\
& =(h+a)^{*}(h+a) Q(x)-(h-a)^{*}(h-a) Q(x) \\
& =h\left((e+a)^{*}(e+a)-(e-a)^{*}(e-a)\right) Q(x) \\
& =h(Q((e+a) x)-Q((e-a) x))=h S(x, y) .
\end{aligned}
$$

Similarly, we obtain that the relation $S(x, h y)=h S(x, y)$ holds. The proof of the theorem is complete.

\section{REFERENCES}

1. J. W. M. Ford, A square root lemma for Banach *-algebras, J. London Math. Soc. 42 (1967), 521-522.

2. S. Kurepa, The Cauchy functional equation and scalar product in vector spaces, Glas. Mat. Fiz.-Astr. 19 (1964), 23-36.

3. __ Quadratic and sesquilinear functionals Glas. Mat. Fiz.-Astr. 20 (1965), 79-92.

4. _ On bimorphisms and quadratic forms on groups, Aequationes Math. 9 (1973), 30-45.

5. V. Pták, Banach algebras with involution, Manuscripta Math. 6 (1972), 245-290.

6. P. Vrbová, Quadratic functionals and bilinear forms, Časopis Pěst. Mat. 98 (1973), 159-161.

UNIVERSITY OF MARIBOR, VEKS̆, RAZLAGOVA 14, 62000 MARIBOR, YUgOSLAVIA 